\title{
A Real Valued Neural Network Based Autoregressive Energy Detector for Cognitive Radio Application
}

\author{
A. J. Onumanyi, ${ }^{1}$ E. N. Onwuka, ${ }^{1}$ A. M. Aibinu, ${ }^{1}$ O. C. Ugweje, ${ }^{2}$ and M. J. E. Salami ${ }^{3}$ \\ ${ }^{1}$ Department of Telecommunication, Federal University of Technology, Minna, Niger State, Nigeria \\ ${ }^{2}$ Digital Bridge Institute, Abuja, Nigeria \\ ${ }^{3}$ Department of Mechatronic Engineering, International Islamic University Malaysia, Kuala Lumpur, Malaysia
}

Correspondence should be addressed to A. J. Onumanyi; adeizal@yahoo.com

Received 31 March 2014; Revised 9 June 2014; Accepted 1 July 2014; Published 29 October 2014

Academic Editor: George Kyriacou

Copyright (C) 2014 A. J. Onumanyi et al. This is an open access article distributed under the Creative Commons Attribution License, which permits unrestricted use, distribution, and reproduction in any medium, provided the original work is properly cited.

\begin{abstract}
A real valued neural network (RVNN) based energy detector (ED) is proposed and analyzed for cognitive radio (CR) application. This was developed using a known two-layered RVNN model to estimate the model coefficients of an autoregressive (AR) system. By using appropriate modules and a well-designed detector, the power spectral density (PSD) of the AR system transfer function was estimated and subsequent receiver operating characteristic (ROC) curves of the detector generated and analyzed. A high detection performance with low false alarm rate was observed for varying signal to noise ratio (SNR), sample number, and model order conditions. The proposed RVNN based ED was then compared to the simple periodogram (SP), Welch periodogram (WP), multitaper (MT), Yule-Walker (YW), Burg (BG), and covariance (CV) based ED techniques. The proposed detector showed better performance than the SP, WP, and MT while providing better false alarm performance than the YW, BG, and CV. Data provided here support the effectiveness of the proposed RVNN based ED for CR application.
\end{abstract}

\section{Introduction}

The energy detector (ED) has been widely proposed for spectrum sensing (SS) in cognitive radio (CR) owing to its design simplicity, fast sensing periodicity, and ability to detect primary user (PU) signal without a priori knowledge of its waveform structure (except for the knowledge of noise statistics) [1-11]. However, the demand for fast sensing and high detection accuracy in CR has revealed particular limitations with the ED. In fast sensing, the ED tends to obtain fewer samples for spectral estimation which leads to reduced resolution and invariably poor detection performance [1215]. Also, it is known that the ED performs poorly in low signal to noise ratio (SNR) conditions and fluctuating noise environments [12-14] which results in increased false alarm rate. These challenges have compelled the need for new improved ED techniques capable of providing better local sensing results at acceptable detection and false alarm rates in both low and high SNR conditions. This serves as the motivation for our research.
Towards developing an ED, several usable spectrum estimation techniques are well-known and these can be broadly divided into parametric and nonparametric techniques. Some examples of these nonparametric techniques include the simple periodogram (SP), Welch periodogram (WP), and the multitaper (MT), while parametric techniques include the Yule-Walker (YW), Burg (BG), and covariance (CV) techniques. Generally, the ED has been widely assumed to be based on the periodogram approach (developed either in time or in frequency domain) and this could be presumably responsible for its poor performance owing to known limitations of the periodogram, for example, large noise variance $[13,14]$. On the other hand, few works have been identified on the use of parametric techniques, particularly the autoregressive (AR) technique, in CR [16-22]. Also, these few works have focused more on its use in spectrum hole prediction rather than as an ED for CR application.

In this work, the focus is not on developing a new spectrum estimation technique but rather on proposing the use of a real valued neural network (RVNN) based autoregressive 
(AR) spectrum estimation technique for ED application in CR. The choice of the RVNN based AR technique was based on its advantage over the popular BG technique in solving the challenge of spectral line splitting at high SNR and large model order conditions [23]. Consequently, it was observed here that such an advantage would lead to an improved ED with less probability of false alarms for CR application. Results of this contribution are presented in Section 5. Also, while authors in [23] developed the RVNN technique and applied it in voice activity detection, in our own work, we propose necessary additions to standardize the approach as a fully functional ED using an empirical threshold estimation technique for CR application. The RVNN based AR model coefficient estimator developed in [23] has never been applied as an ED for CR application; hence, its application was considered here to exploit its inherent advantage. Therefore, once developed as a functional ED, it was tested using simulated data particularly in low and high SNR conditions and varying model order values as described in Section 4 and results obtained with corresponding implications are presented in Section 5. Appropriate conclusions are drawn in Section 6.

\section{Brief Review of Energy Detector Techniques}

Energy detectors (ED) sense the presence or absence of $\mathrm{PU}$ signals by estimating the energy content of a specified band using the power spectral density (PSD) measurements and comparing with predetermined thresholds $[12,14]$. This aids the CR device in vacating PU occupied bands to avoid interference and use vacant bands to improve spectrum utilization. Figures 1(a) and 1(b) provide a representation of this process. Towards this goal, signals above the threshold typically signify PU presence, $H_{1}$, and below threshold as noise, $H_{0}$. These hypotheses $H_{0}$ and $H_{1}$ are typically represented as

$$
\begin{gathered}
H_{0}: x(n)=w(n) \\
H_{1}: x(n)=s(n)+w(n),
\end{gathered}
$$

where $w(n)$ represents samples of additive white Gaussian noise (AWGN), $s(n)$ denotes samples of PU signal, and $x(n)$ denotes samples of received signal at the CR input considering a perfect channel.

EDs are typically developed to decide on these hypotheses either in time or in frequency domain. In time domain, it consists of an input noise prefilter, a magnitude square law device, an integrator, and detector as shown in Figure 2. This approach has been used in several works [12-15] and wellknown for its simplicity and low design and development cost. However, it is based on the super heterodyne technique which is limited by its slow voltage ramping response time [6]. Such a delay might be intolerable for CR operation. Another approach is to realize the $\mathrm{ED}$ in the frequency domain using the well-known periodogram as shown in Figure 3. This provides an opportunity to achieve full digitization of the detection process; however, this approach constrains the sensing bandwidth owing to known limitations of typical fast Fourier transform (FFT) realizations [8].

Another method identified for the ED in CR is the Welch periodogram (WP) approach [24]. Particularly, in [25], performance analysis was carried out on the use of WP in Rayleigh fading channels while $[26,27]$ used WP for OFDM systems in CR. These authors observed that WP operates well for narrowband sensing; however, they conclude that the approach is limited by the excessive variance around the true mean PSD value. This increases the probability of false alarm of the system. Another approach using the Multitaper (MT) technique was proposed in [28] with basic descriptions provided from filter-bank theory point of view. This technique was extended in [29] for signal detection in $\mathrm{CR}$ and results were compared with the time domain based ED. It was observed that MT performed better in low SNR conditions under Raleigh fading channels. This was based on the technique's dependence on both magnitude and phase of received samples. Also, [30] provided an improved MT method for CR application in which a recursive method to improve the accuracy of estimates while keeping real-time properties uncompromised was proposed. Authors compared their technique with the SP and ordinary MT and observed better performance for their improved MT.

On the other hand, the use of autoregressive (AR) methods has been sparsely pursued, especially for energy detection in CR. The AR methods present a class of techniques popularly classified as parametric techniques. Though not widely used in CR, we observed that [19-21] proposed AR for spectrum hole detection in CR using time series forecasting. Authors reported on the performance of the proposed approach; however, it remains unclear how well such an approach would perform in typical deployment conditions. Also, authors in $[21,22]$ used particle and Kalman filtering for spectrum availability forecasting. In [17], authors presented a comparison of SP and Yule-Walker (YW) AR techniques and argued that better performance could be achieved by the YW if large model orders are considered. However, most of these applications have not considered AR application for ED in $\mathrm{CR}$, and hence, it remains unknown how well such techniques would perform if employed. Consequently, we considered the use of a real valued neural network (RVNN) AR model estimator [23] to develop a detector for CR application. This approach provides data reported in [23] which was leveraged upon here to develop an improved ED for CR. Consequently, it is the focus here to develop, evaluate, and analyze its performance for CR application.

\section{Development of the Real Valued Neural Network Based Energy Detector}

3.1. An Overview of the RVNN Based Autoregressive Model Estimation Technique. The working principles of the real valued neural network (RVVN) autoregressive (AR) coefficient estimation technique were based on [23]. Thus, we present here only specific details needed to develop the ED for CR application; however, for further details, we refer to [23]. 


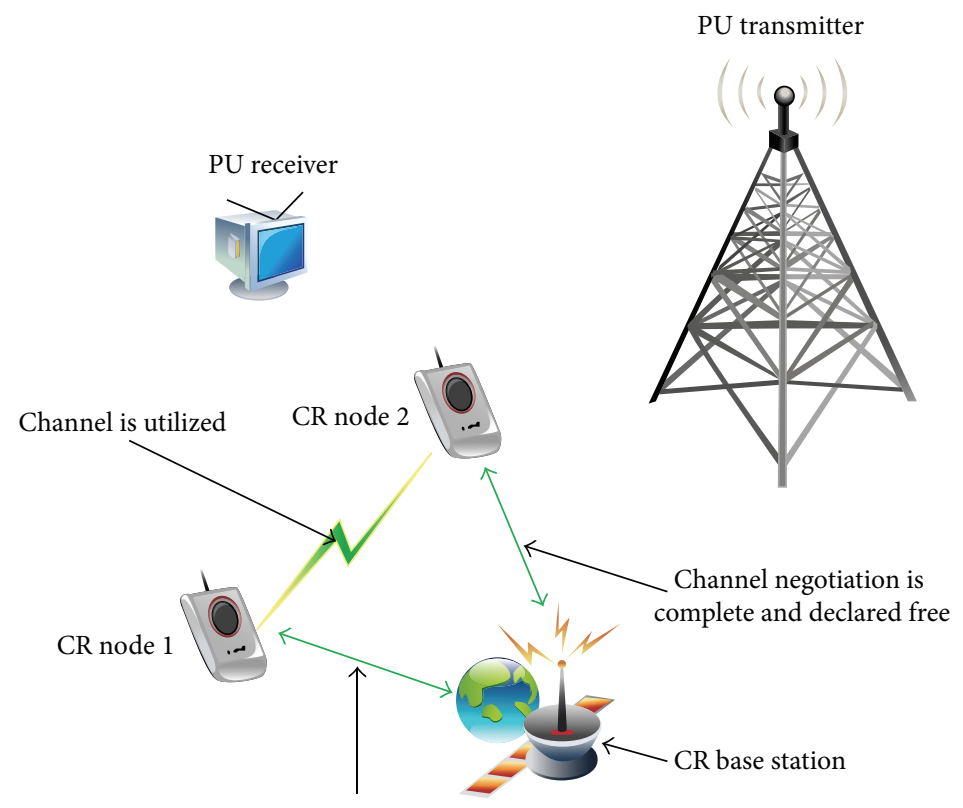

Channel negotiation is complete and declared free

(a)

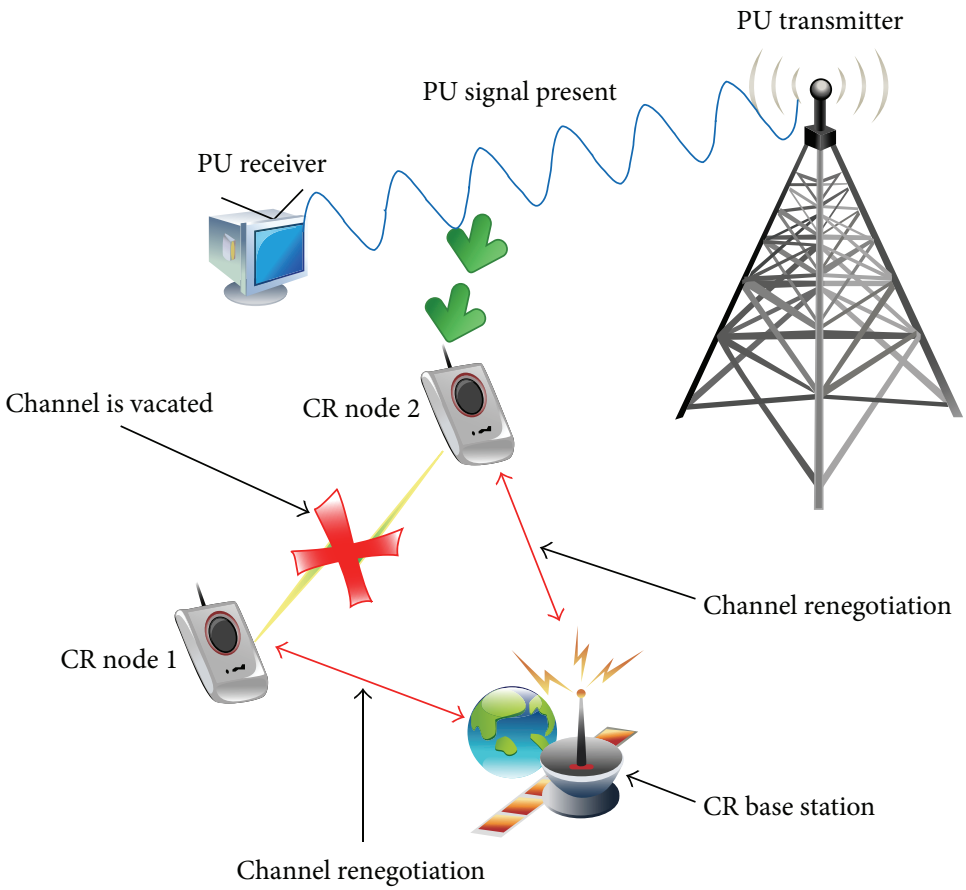

(b)

Figure 1: (a) Absence of PU signal permitting CR nodes to negotiate and utilize free band. (b) Presence of PU signal triggers CR nodes to renegotiate and vacate band.

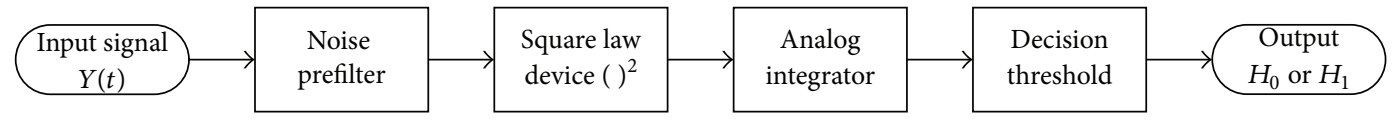

FIgURE 2: Energy detection in time domain. 


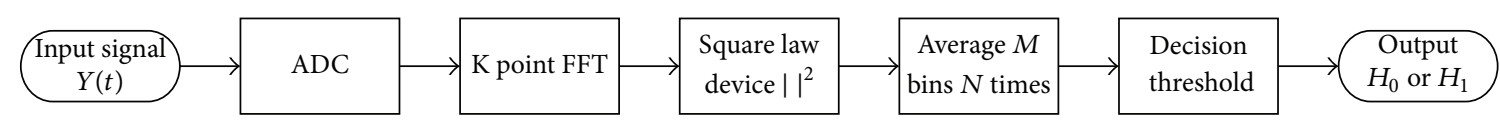

FIGURE 3: Energy detection using the simple periodogram (frequency domain approach).

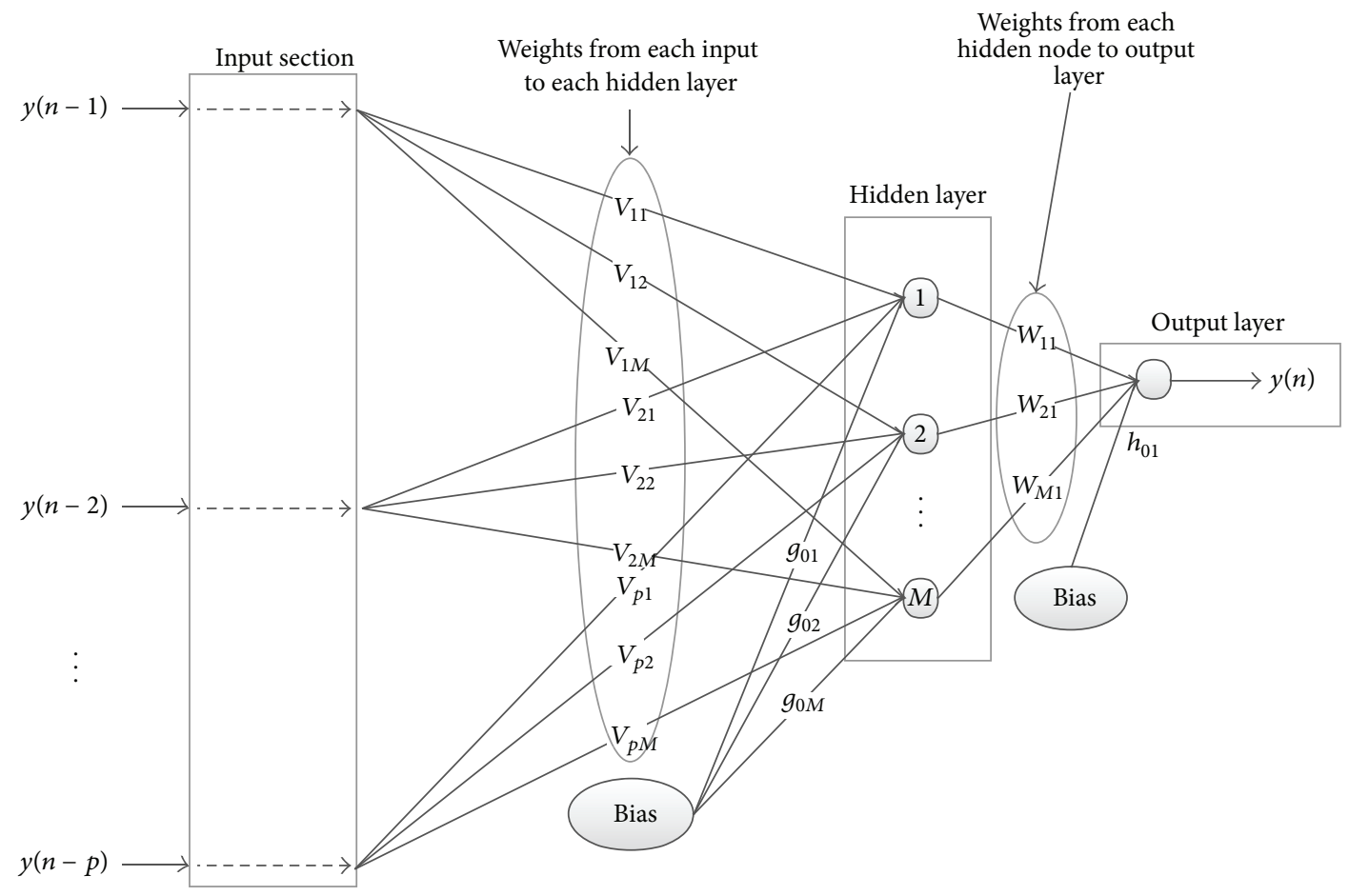

FIgURE 4: A two-layered RVNN network system.

It is known that an $\mathrm{AR}$ system driven by white noise $x(n)$ produces an output sequence $y(n)$ given as

$$
y(n)=-\sum_{k=1}^{P} a_{k} y(n-k)+b_{0} x(n),
$$

where $a_{k}, 1 \leq k \leq P$ and $b_{0}$ denotes the model coefficients and $P$ is the model order. By taking the $z$-transform of (3) and rearranging the terms therein, the transfer function $H(z)$ of the AR-system can be obtained as

$$
H(z)=\frac{Y(z)}{X(z)}=\frac{b_{0}}{1+\sum_{k=1}^{P} a_{k} z^{-k}} .
$$

By assigning $b_{0}=1, z=e^{-j w}$ and taking the square of (4), we obtain the SNR as

$$
\mathrm{SNR}=\left|\frac{Y(j w)}{X(j w)}\right|^{2}=\frac{1}{\left|1+\sum_{k=1}^{P} a_{k} e^{-j w k}\right|^{2}} .
$$

To estimate the model coefficient $a_{k}$, we used a twolayered RVNN as shown in Figure 4.

By observing Figure 4, the output sequence of the RVNN system can be obtained as

$$
y(n)=\alpha \mathbf{F}\left(\sum_{l=1}^{M} w_{l 1} \theta_{l}+h_{01}\right)
$$

where $\mathbf{F}$ denotes the representation of a linear transfer function, $M$ is the number of neurons in the hidden layer, $w_{l 1}$ represents the weight connecting node $l$ in the hidden to output layer, $h_{01}$ is the bias term of the output neuron, $\theta_{l}$ denotes the output of the hidden node $l$, and $\alpha$ is the adaptive coefficient of the linear output activation function. The hidden node output $\theta_{l}$ is obtained as

$$
\theta_{l}=\beta_{l} \mathbf{F}\left(\sum_{k=1}^{P} v_{k l} y(n-k)+g_{0 l}\right)
$$

where $v_{k l}$ is the weight connecting input nodes $k$ to hidden node $l, g_{0 l}$ is the bias of the hidden node, and $\beta_{l}$ is the adaptive coefficient of the hidden node linear transfer function. By using linear transfer activation functions in both hidden and output layer, that is, $\mathbf{F}(\cdot)$ is linear, and substituting (7) into (4), the output sequence is obtained as

$y(n)$

$$
=\alpha \mathbf{F}\left(\sum_{l=1}^{M} w_{l 1}\left(\beta_{l} \mathbf{F}\left(\sum_{k=1}^{P} v_{k l} y(n-k)+g_{0 l}\right)\right)+h_{01}\right) .
$$




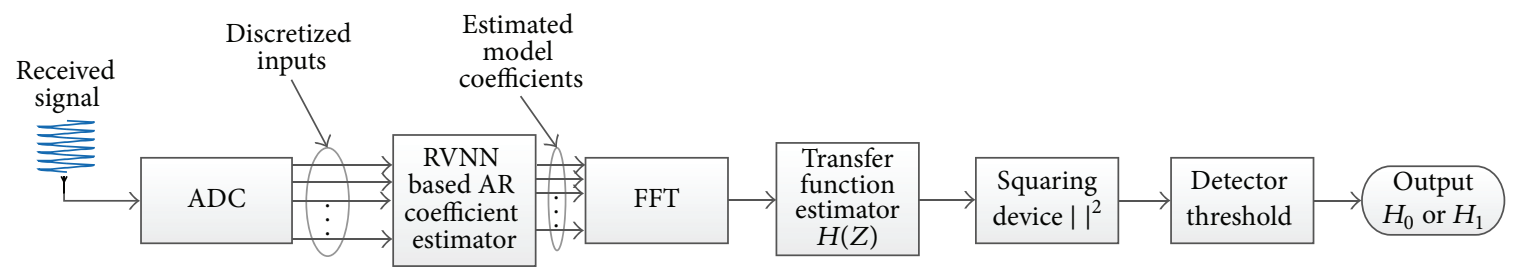

Figure 5: The newly proposed RVNN based ED.

By rearranging terms in (8) and comparing with (4), the model coefficients $a_{k}$ can be expressed as

$$
a_{k}=\alpha \sum_{l=1}^{M} w_{l 1} \beta_{l} v_{k l} \text {. }
$$

Thus, the AR model coefficients can be estimated from the synaptic weights and coefficients of the adaptive activation function of a properly trained two-layered RVNN. Furthermore, by assuming that the model order $P$ is known $a$ priori, then the number of neurons in the hidden layer can be determined by using the priori data length information at the input. To formulate the necessary constraints for proper evaluation of the system, the solution to a set of linear equations that gives the best performance over the $a$ priori fixed model order was obtained. We obtained this by considering a data set of length $N$, for which the required number of training data set $L$ was obtained as

$$
L=N-P+1 \text {. }
$$

The required number of training equations $N_{\text {eq }}$ is expressed as

$$
N_{\mathrm{eq}}=P \times L
$$

and for $M$ hidden nodes, the number of unknowns for the network was obtained as

$$
N_{\text {un }}=(P+1) M+M+1 .
$$

It is known from [23] that the case of $N_{\text {eq }} \gg N_{\text {un }}$ produces better results with respect to the unseen data. Therefore, by putting (11) and (12) into the inequality $\left(N_{\text {eq }} \gg N_{\text {un }}\right)$, we obtained the optimum number of neurons $M$ to satisfy

$$
M \ll \frac{(P \times L)-1}{P+2} .
$$

With these necessary parameters well established, the algorithm used for the RVNN system is as follows.

(1) We normalized the input data sequence to band-limit the acquired signal using the $Z$-score normalization technique. Other techniques that could be used are the Min-Max, sigmoidal, or unitary data normalization techniques.

(2) The data were formatted using frame blocking and appropriate windowing of data sequencing.
(3) The optimum number of neurons was determined using (13).

(4) The model coefficient was estimated using (9).

(5) Finally, training of the RVNN system was done using the back propagation (BP) supervised learning algorithm.

3.2. The RVNN Based Energy Detector. With the RVNN model estimator in place, we developed the RVNN based ED using the proposed schematic of Figure 5. This was achieved by generating the power spectral density (PSD) of the system using (5) and introducing a well-designed detector based on an empirical threshold estimation technique (details of method in Section 4.2). An overview of the design process is as follows.

(1) A simple analogue-digital-converter (ADC) was used for digitization. Here the number of samples was determined using the known Nyquist rate of $8 \mathrm{KHz}$ for a maximum bandspan of $4 \mathrm{KHz}$ for wideband simulation and $100 \mathrm{~Hz}$ for narrowband simulation.

(2) The fast Fourier transform (FFT) was used for the frequency response estimation. Appropriate zero padding was employed for cases of fewer samples for FFT operation.

(3) The transfer function estimator was realized using the frequency response vector obtained from appropriate adaptive filtering of the FFT samples.

(4) A simple square law device was introduced for performing the squaring operation.

(5) The detector analyzer was realized using empirically deduced thresholds (details in Section 4.2).

(6) Our proposed detector of Figure 5 can be used for CR application and details of methods used for its analysis are presented in the next section.

\section{Method of Modelling and Simulation}

In this section, we provide details of simulation parameters and analytical models used for evaluating the performance of our proposed detector and other methods for comparison.

4.1. Hypothesis Testing. Additive white Gaussian noise (AWGN) with zero mean and unit variance was used and the probability of false alarm $P_{\mathrm{FA}}$ conditioned for the null 


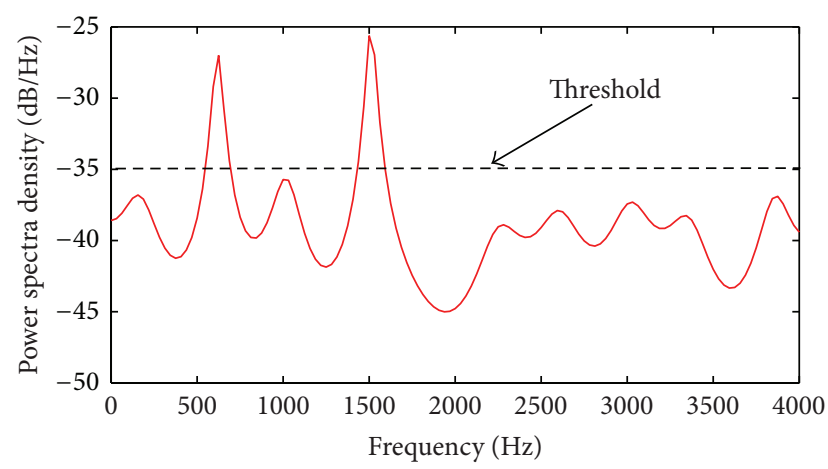

FIGURE 6: Wideband spectrum sensing of $4 \mathrm{KHz}$ showing two PU signals at $600 \mathrm{~Hz}$ and $1.5 \mathrm{KHz}$ in AWGN (occupancy $=10 \%$ ).

hypothesis (1) was obtained using the expression for large time-bandwidth product given in [31]. The $P_{\mathrm{FA}}$ for noise samples distributed according to a Gaussian variate $N(2 T W, 4 T W)$ is given as [31]

$$
\begin{aligned}
P_{\mathrm{FA}} & =\frac{1}{\sqrt{8 \pi T W}} \int_{V_{T}}^{\infty} \exp \left[-\frac{(y-2 T W)^{2}}{8 T W}\right] d y \\
& =\frac{1}{2} \operatorname{erf} c\left[\frac{V_{T}-2 T W}{2 \sqrt{2} \sqrt{T W}}\right],
\end{aligned}
$$

where $T$ denotes the observation interval, $W$ the bandwidth being sensed, $V_{T}$ the threshold, and $T W$ the time-bandwidth product. These were translated appropriately as follows: $T W$ is known as the total number of samples $N$ (at Nyquist rate), $2 T W$ is the sample mean $\widehat{\mu}$ of the noise PSD, and $4 T W$ is the sample variance $\widehat{\sigma}^{2}$ of the noise PSD (the noise power). Hence, we rewrite (15) as

$$
P_{\mathrm{FA}}=\frac{1}{2} \operatorname{erf} c\left[\frac{V_{T}-\widehat{\mu}}{\sqrt{2 \widehat{\sigma}^{2}}}\right] .
$$

We note that the particular use of (15) according to [31] was based on the condition of a large time-bandwidth product, meaning large sample number. Next, to estimate the probability of detection $P_{D}$, we considered a modulated discrete signal $S(n T)$ given as

$$
S(n T)=A(t) \cos \left(2 \pi f_{c} n T\right) \quad \forall n=0,1, \ldots, N-1,
$$

where $A(t)$ represents the expression for the modulating signal, $f_{c}$ the carrier frequency, $N$ the total number of samples, and $T$ the sampling time.

For examination of the effect of wideband sensing, we considered two PU signals with equal amplitudes $A_{1}=$ $A_{2}=10 \mathrm{~dB}$, and frequencies $f_{c 1}=600 \mathrm{~Hz}, f_{c 2}=1500 \mathrm{~Hz}$ estimated over a relatively wide sensing span of $4 \mathrm{~Hz}$ using a wideband occupancy of $10 \%$ as shown in Figure 6 . We note that though low frequencies were used, the setup could easily apply to high frequency bands with the same concept being applicable.

For narrowband sensing, a typical PU signal of bandwidth $W=60 \mathrm{~Hz}$ was simulated for a narrowband sweep of

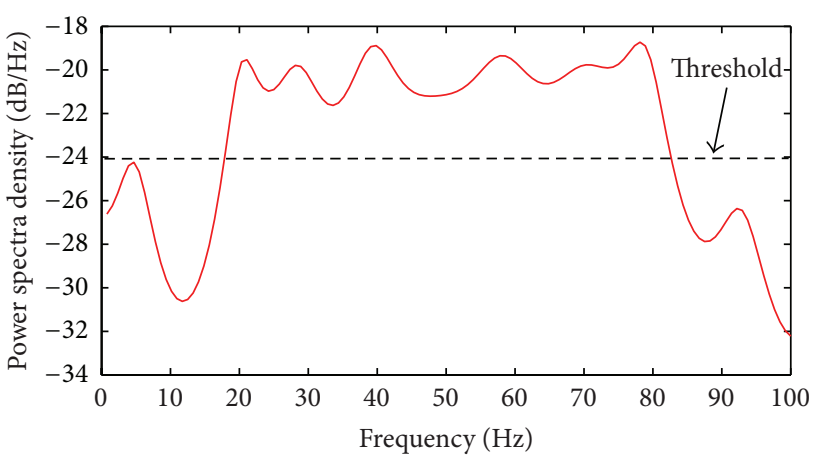

Figure 7: Narrowband spectrum sensing of $100 \mathrm{~Hz}$ showing PU bandwidth of $60 \mathrm{~Hz}$ and guardbands of $20 \mathrm{~Hz}$ on both sides.

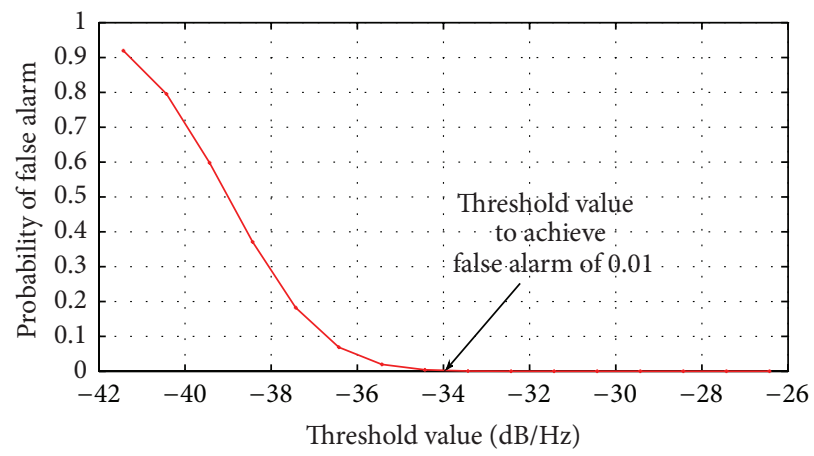

FIGURE 8: Probability of false alarm for different thresholds using the RVNN based ED in wideband sensing.

$100 \mathrm{~Hz}$ as shown in Figure 7 using the RVNN based ED. These input data were kept constant for all simulation conditions and other EDs compared herein. Then the $P_{D}$ was estimated using [31]

$$
P_{D}=\frac{1}{2} \operatorname{erf} c\left[\frac{V_{T}-2 T W-\lambda}{2 \sqrt{2} \sqrt{T W+\lambda}}\right],
$$

where $\lambda$ denotes the SNR for the estimated spectrum.

4.2. Threshold Estimation. An empirical threshold selection method was employed here and $P_{\mathrm{FA}}$ for each threshold was estimated using (16). This was done by varying the threshold values $V_{T}$ over the noise PSD and Figure 8 provides the result obtained using the RVNN based ED in wideband sensing. By comparing the threshold line in Figure 6 with Figure 8, it can be easily observed that at a threshold of $-35 \mathrm{~dB} / \mathrm{Hz}$, less noise samples crossed the threshold (Figure 6) resulting in $P_{\mathrm{FA}}<$ 0.01 as seen in Figure 8. This provided a suitable threshold for estimating the detector performance.

\section{Results and Discussion}

5.1. Varying AR Model Order on RVNN Based ED. The effect of varying AR model order on the performance of the RVNN based ED was analyzed by inspecting the outcome of different ROCs under varying model order values. This examination 


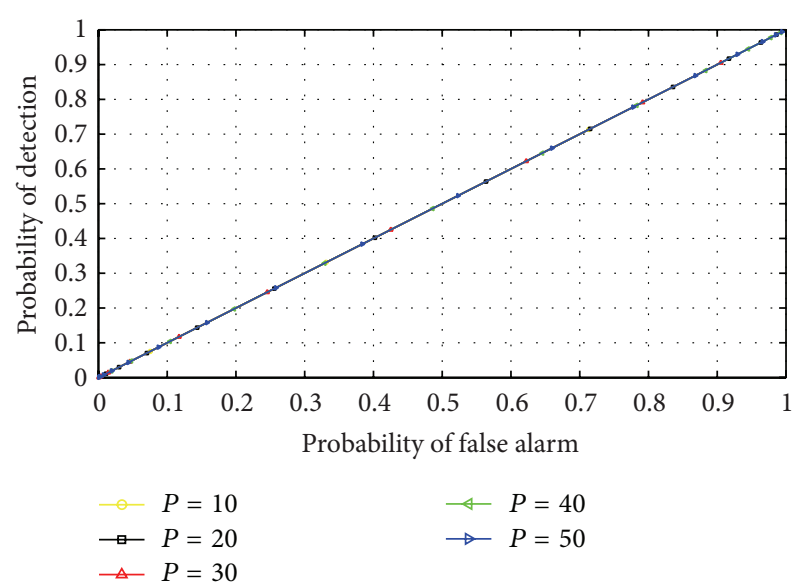

FIGURE 9: ROC for RVNN based ED in narrowband sensing under low SNR $(0 \mathrm{~dB})$ and sample number $N=250$.

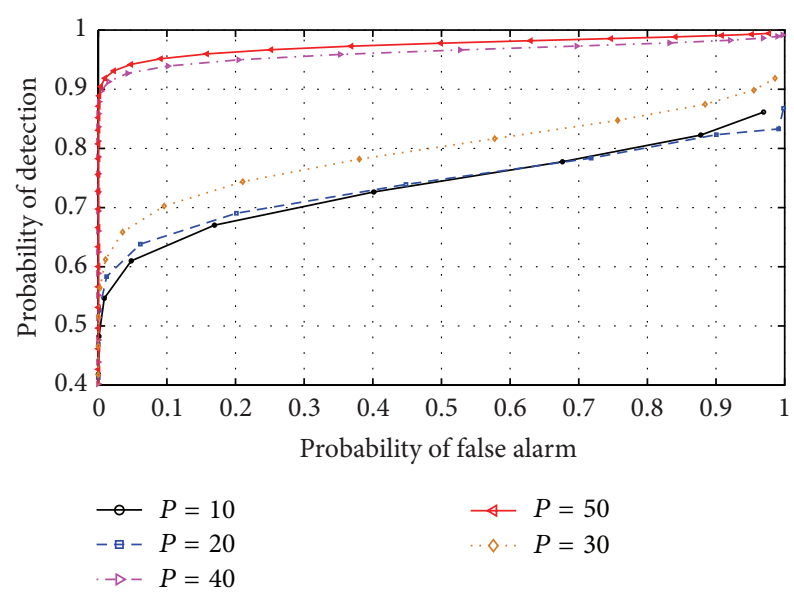

FIGURE 10: ROC for RVNN based ED in narrowband sensing under high SNR $(10 \mathrm{~dB})$ and sample number $N=250$.

was essential to aid in the choice of optimum model order for CR operation. Furthermore, this effect was investigated in both narrow and wideband sensing to determine which scenario best supports the detector. The detector was also examined in low $(0 \mathrm{~dB})$ and high $(10 \mathrm{~dB})$ SNR conditions, respectively. It can be observed from Figures 9 and 11 that the ROCs for different orders remained approximately equal for low SNR in both narrow and wideband sensing. This confirmed that, at low SNR, the PU signal is totally submerged in noise and cannot be differentiated by the use of energy levels alone resulting in $P_{\mathrm{FA}} \approx P_{D}$. This also indicated that irrespective of the approach, the ED typically performs poorly in low SNR conditions. However, in narrowband sensing, high SNR, and varying model order of Figure 10, the RVNN based ED was observed to perform well, particularly for model order $P=50$. We also observed that the difference in performance becomes practically negligible for $P \geq 40$. Hence, a minimum order of $P=40$ was observed to achieve high detection performance. For the case of wideband sensing, high SNR, and varying model

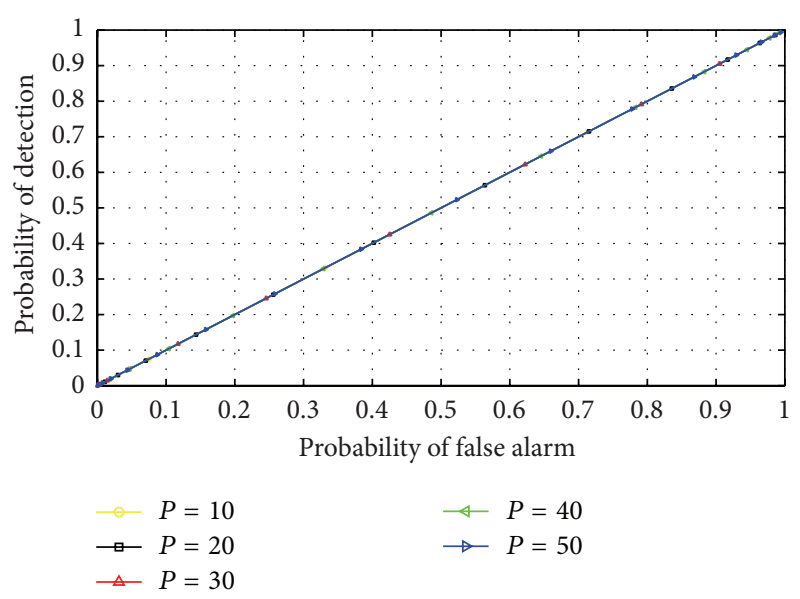

FIGURE 11: ROC for RVNN based ED in wideband sensing under low SNR (0 dB) and sample number $N=250$.

order, Figure 12 revealed a reverse in performance for the model order values investigated here. In this case, model order $P=50$ was observed to perform poorly while $P=$ 20 provided better performance. We note that this outcome was based on the increased spectral leakage at lower order which resulted in more samples crossing the threshold than at $P=50$. This effect is evident owing to the large spectrum considered under wideband sensing as compared to the percentage occupancy of the signal within the band. Hence, it can be inferred that the probability of false alarm would consequently increase for such lower model orders than the higher orders, thus making it uncertain to strictly rely on the results of an ROC curve alone. However, this observation would be further studied and analysed in future works. By comparison, it was observed that narrowband sensing produces better performance than wideband for the RVNN based ED. This can be conclusive if the sensing bandwidth of the narrowband detector corresponds typically to the transmission bandwidth of the PU signal. The implication of these results means that to use the RVNN based ED for CR, narrowband sensing at $P=50$ for short data length (fast sensing time) will be ideal to produce the best detection performance.

5.2. Varying Sample Number on RVNN Based ED. To examine this effect, a fixed choice of AR model order $P=50$ was selected for narrowband sensing while $P=20$ was chosen for wideband sensing. This ensured that the best model order values were used to examine the effect of sensing time, that is, varying sample number. Here we examined only for the high SNR case $(10 \mathrm{~dB})$ knowing that performance remains the same in low SNR conditions (seen in Figures 9 and 11). By observing Figure 13, it was seen that detection performance of RVNN based ED improved with increase in sample number $N$. However, this increase became negligible for $N \geq 2000$. We note that this performance was achieved for $P=50$. Furthermore, by using $P=20$ for wideband sensing, Figure 14 revealed that high performance was sustained even 


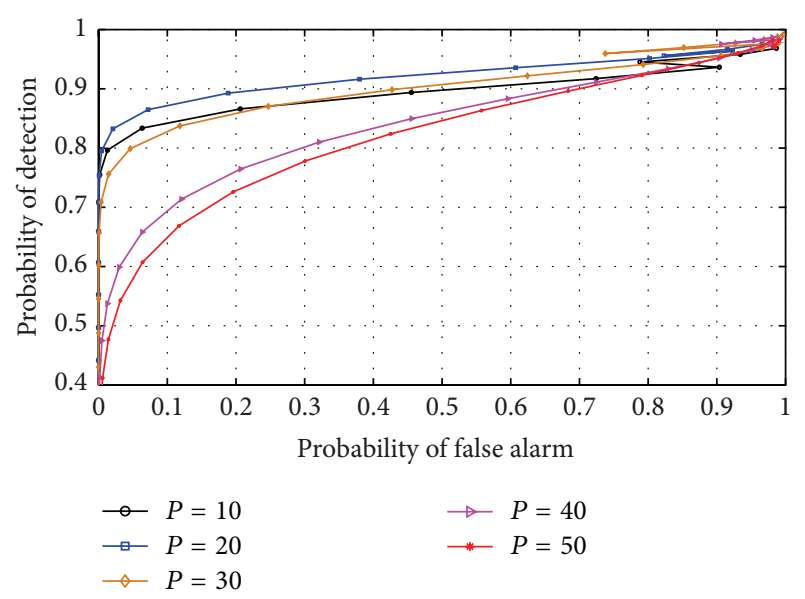

FIGURE 12: ROC for RVNN based ED in wideband sensing under high SNR $(10 \mathrm{~dB})$ and sample number $N=250$.

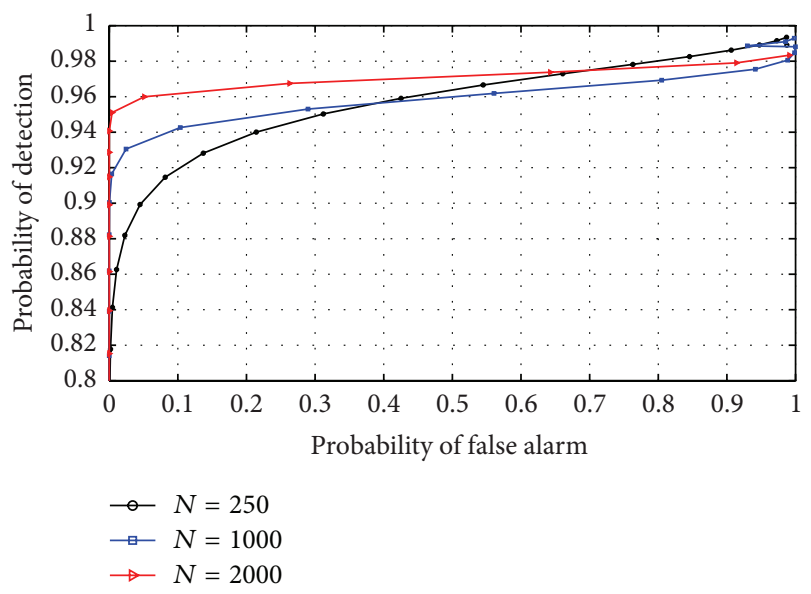

FIGURE 13: ROC for RVNN based ED in narrowband sensing under high SNR $(10 \mathrm{~dB})$ and $P=50$.

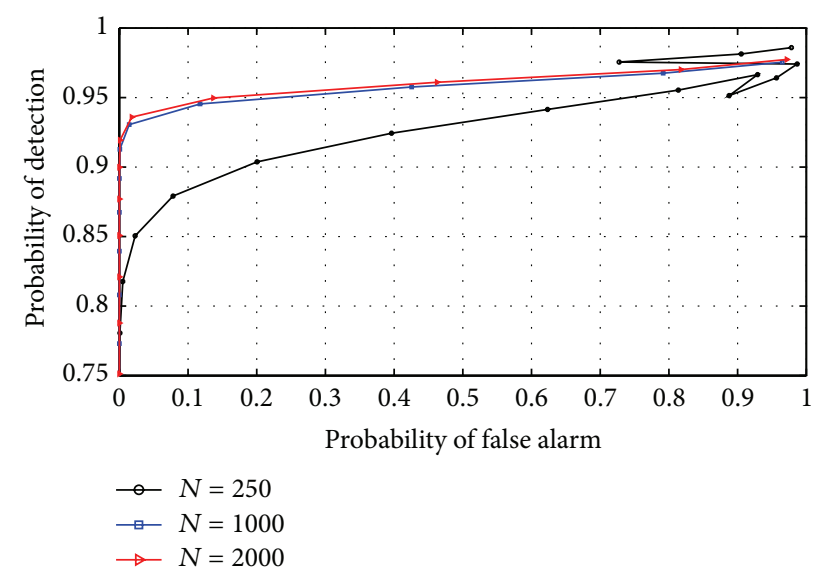

FIGURE 14: ROC for RVNN based ED in wideband sensing under high SNR $(10 \mathrm{~dB})$ and $P=20$.

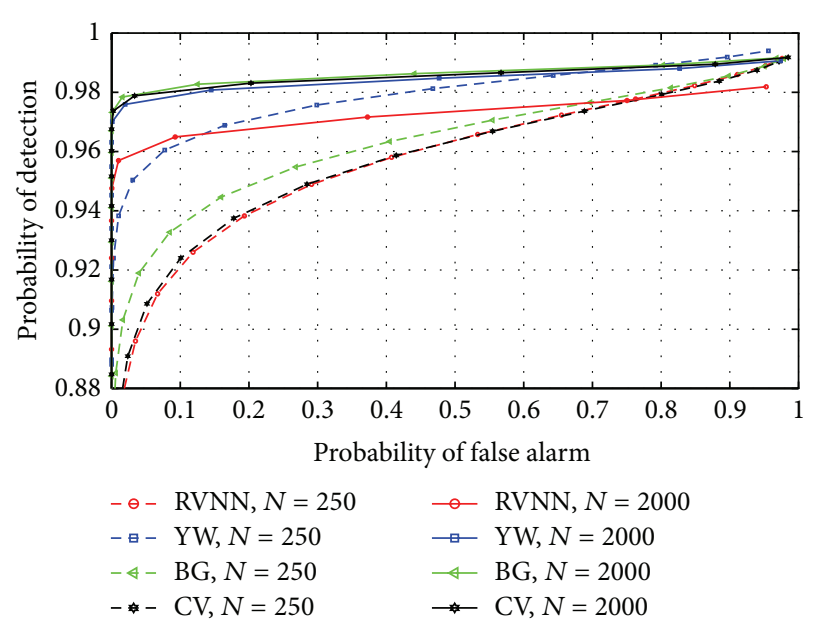

FIGURE 15: ROCs compared for different parametric based EDs using $P=50$ in narrowband sensing.

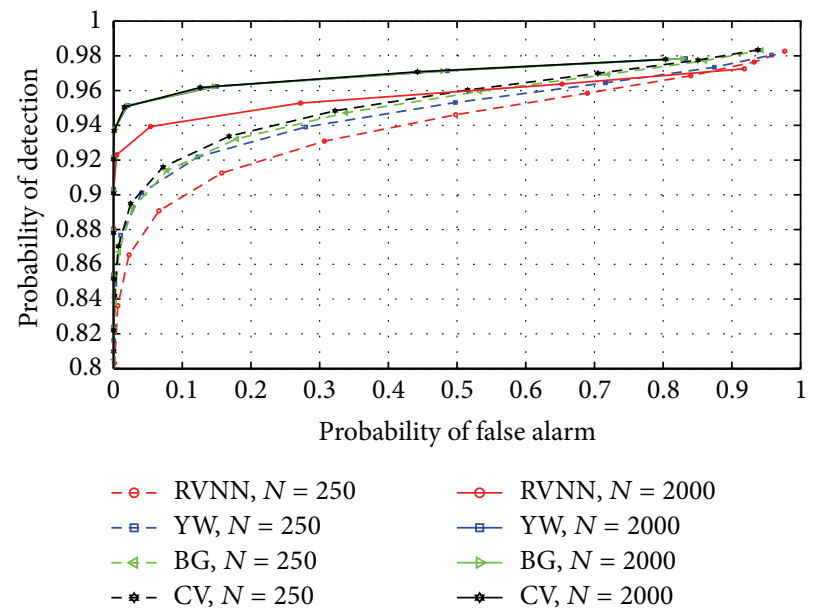

FIGURE 16: ROCs compared for different parametric based EDs for $P=20$ in wideband sensing.

with a slight performance drop in comparison to narrowband sensing. This implied that to use the RVNN based ED for fast sensing in $\mathrm{CR}$, narrowband sensing for $P=50$ remains ideal.

5.3. Comparison with Other Parametric Based ED Techniques. The RVNN based ED was compared with the Yule-Walker (YW), Burg (BG), and covariance (CV) based approaches. Narrowband sensing was examined using model order $P=$ 50 while $P=20$ was chosen for wideband sensing. Figure 15 revealed a close comparative performance between all techniques for fasting sensing time $(N=250)$. Further comparison was conducted for long sensing time $(N=2000)$ and once again, performance level was close. However, it was observed that performance increased for all techniques with increase in sample number. This implied that long sensing time improved the performance of the RVNN based ED and other techniques. For wideband sensing, Figure 16 revealed a close detection performance between RVNN based ED and 


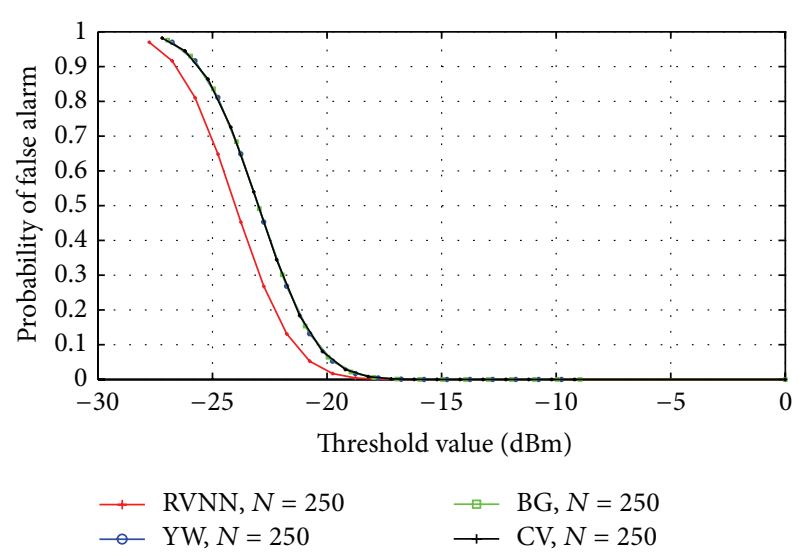

FIGURE 17: Comparison of probability of false alarm for different parametric based techniques for the case of narrowband sensing at $N=250$.

other parametric techniques; however, a $2 \%$ drop in detection performance was observed with respect to BG, YW, and CV. Comparatively, the RVNN based ED produced similar detection performance to acceptable specifications $\left(P_{D}>0.9\right.$ at $P_{\mathrm{FA}}=0.1$ ) along with other techniques. In addition, it is shown in Figure 17 that the proposed technique provides a $10 \%$ average reduction in $P_{\mathrm{FA}}$ at thresholds below $-20 \mathrm{dBm}$ than $\mathrm{YW}, \mathrm{BG}$, and $\mathrm{CV}$. This revealed a possible $1 \mathrm{~dB}$ reduction in threshold level to achieve $P_{\mathrm{FA}}=0.1$ as compared to other parametric techniques studied here. This means that the RVNN based ED provides better sensitivity than YW, BG, and CV based approaches. Also, we note that YW, BG, and $\mathrm{CV}$ have tendencies to overestimate the magnitude response of a signal which resulted in the observed better ROC performance of $\mathrm{YW}, \mathrm{BG}$, and $\mathrm{CV}$ over the proposed technique in Figures 15 and 16. Nevertheless, higher SNR estimation and consequently improved ROC can be achieved for the RVNN based ED by including appropriate amplifiers after the square law device in Figure 5. On the other hand, false alarms are more difficult to address and cannot be solved by simple amplification as proposed because both noise and signals will be amplified accordingly, except if noise reduction techniques are used. Consequently, reducing $P_{\mathrm{FA}}$ could depend more on threshold selection or on the underlying nature of the detector. Therefore, Figure 17 emphasizes a critical advantage of the RVNN based ED over other parametric techniques especially in providing an inherent capacity to reduce the $P_{\mathrm{FA}}$ level and prevent possible interference to primary user (PU) signals. This is a highly desirable feature of any good detector for CR application.

5.4. Comparison with Nonparametric Techniques. Finally, the RVNN based ED was compared with nonparametric techniques such as the simple periodogram (SP), Welch periodogram (WP), and multitaper (MT). For narrowband sensing, Figure 18 revealed that the RVNN based ED performed better than the nonparametric techniques compared here except for WP which performed slightly better. This was due to the high variance estimation obtained in the WP

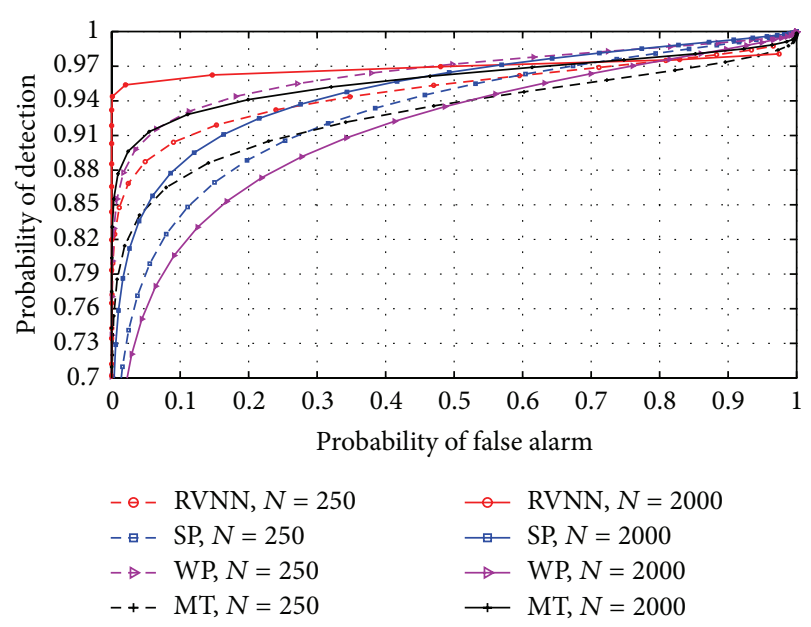

FIGURE 18: ROCs compared between RVNN based ED and other nonparametric techniques in narrowband for $P=50$.

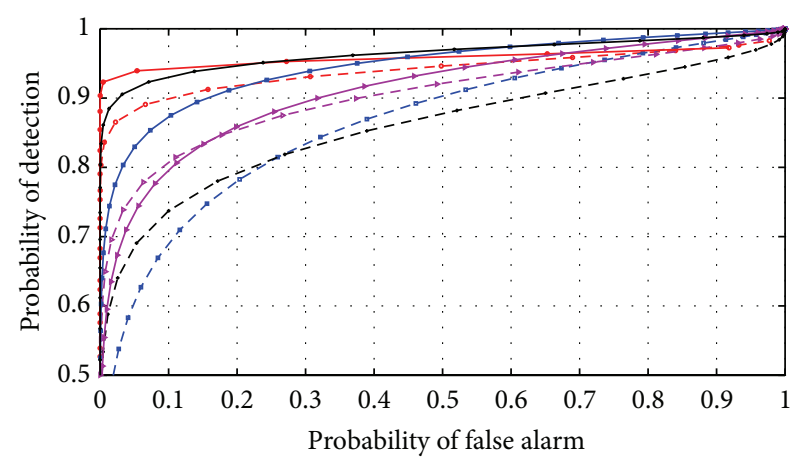

$$
\begin{aligned}
& -\theta-\text { RVNN, } N=250 \quad \rightarrow \quad \text { RVNN, } N=2000 \\
& -\because-\mathrm{SP}, N=250 \quad \rightarrow \mathrm{SP}, N=2000 \\
& \rightarrow-\text { WP, } N=250 \quad \rightarrow \text { WP, } N=2000 \\
& \text {-+- MT, } N=250 \quad \longrightarrow \text { MT, } N=2000
\end{aligned}
$$

FIGURE 19: ROCs compared between RVNN based ED and other nonparametric techniques for wideband sensing for $P=20$.

which made more noise samples to cross the threshold than in the RVNN based ED. In wideband sensing, Figure 19 revealed that the RVNN based ED performed better than other nonparametric techniques.

\section{Conclusion}

In this paper, a real valued neural network (RVNN) based energy detector (ED) has been proposed, simulated, and analyzed. The choice of the RVNN method for estimating the coefficients of an autoregressive (AR) system was based on its inherent ability to solve the challenge of line splitting and spectral shifting as reported in [23]. Thus, our developed RVNN based ED was examined using different simulation parameters for different sensing conditions. We observed that, for model order $P=50$, the proposed detector provided a high detection performance in narrowband sensing and 
similar performance in wideband sensing for $P=20$. Furthermore, the detector was compared with other parametric based techniques like the Yule-Walker (YW), Burg (BG), and covariance $(\mathrm{CV})$ methods. A close performance was achieved between our proposed ED and others with less than 5\% drop in ROC performance observed with respect to YW, $\mathrm{BG}$, and $\mathrm{CV}$. However, a $10 \%$ reduction in false alarm was observed for the RVNN based ED over other parametric techniques. This result presents an important advantage in using the proposed technique for CR application because techniques with less false alarms are highly desirable. Finally, it was compared with some nonparametric techniques like the simple periodogram (SP), Welch periodogram (WP), and the multitaper (MT). Our proposed detector provided a $10 \%$ detection performance gain over these techniques except in narrowband and fast sensing case where WP provided about $2 \%$ detection increase than the RVNN based ED. This was attributed to the high estimated noise variance level which consequently resulted in increased false alarm rates in WP. The study reported here indicates that the RVNN based ED provides a new option for spectrum sensing in CR. However, we note that attention was not given here to the degree of design complexity with respect to choice of higher model order. Though it has been shown in [17] that computational demand in terms of number of multiplications is less than the periodogram, likewise for the storage demand, no closed form model exists to describe complexity for higher model order. This might not necessarily affect detection or false alarm performance but might affect timing performance. However, this remains to be studied and provides an opportunity for future research investigations in the field of AR application in CR.

\section{Conflict of Interests}

The authors declare that there is no conflict of interests regarding the publishing of this paper.

\section{Acknowledgment}

This work was supported under the University Board of Research (UBR) grant award from the Federal University of Technology, Minna, Nigeria.

\section{References}

[1] M. López-Benítez and F. Casadevall, "On the spectrum occupancy perception of cognitive radio terminals in realistic scenarios," in Proceedings of the 2nd International Workshop on Cognitive Information Processing (CIP '10), Special Session on NEWCOMTF, pp. 99-104, Elba, Italy, June 2010.

[2] A. J. Onumanyi, E. N. Onwuka, O. Ugweje, and M. J. E. Salami, "Spectroscopic measurements and analysis of frequency occupancy: a case study of Minna, Niger State," in Proceedings of the 3rd Biennial Engineering Conference, pp. 30-35, Federal University of Technology, Minna, Nigeria, 2013.

[3] R. Urban, T. Kriz, and M. Cap, "Indoor broadband spectrum survey measurements for the improvement of wireless systems," in Proceedings of the Progress in Electromagnetics Research
Symposium (PIERS '13), pp. 376-380, Taipei, Taiwan, March 2013.

[4] M. N. Mehdawi, K. Riley, A. Paulson, M. Fanan, and M. Ammar, "Spectrum occupancy survey in HULL-UK for cognitive radio applications: measurement \& analysis," International Journal of Scientific \& Technology Research, vol. 2, no. 4, pp. 231-236, 2013.

[5] D. Datla, A. M. Wyglinski, and G. J. Minden, "A spectrum surveying framework for dynamic spectrum access networks," IEEE Transactions on Vehicular Technology, vol. 58, no. 8, pp. 4158-4168, 2009.

[6] J. Polson and B. A. Fette, "Cognitive techniques: position awareness," Cognitive Radio Technology, pp. 265-288, 2009.

[7] B. Wang and K. J. R. Liu, "Advances in cognitive radio networks: a survey," IEEE Journal on Selected Topics in Signal Processing, vol. 5, no. 1, pp. 5-23, 2011.

[8] Y. Hur, J. Park, W. Woo, J. S. Lee, K. Lim, and C. Lee, "A cognitive radio (CR) system employing a dual-stage spectrum sensing technique: A Multi-Resolution Spectrum Sensing (MRSS) and a Temporal Signature Detection (TSD) technique," in Proceedings of the IEEE Global Telecommunications Conference (GLOBECOM '06), pp. 1-5, San Francisco, Calif, USA, December 2006.

[9] M. Nekovee, "Mechanism design for cognitive radio networks," in Proceedings of the Conference on Complexity in Engineering (COMPENG '10), pp. 12-17, Rome, Italy, February 2010.

[10] E. Hossain, D. Niyato, and D. I. Kim, "Evolution and future trends of research in cognitive radio: a contemporary survey," Wireless Communications and Mobile Computing, 2013.

[11] I. F. Akyildiz, W. Lee, M. C. Vuran, and S. Mohanty, "NeXt generation/dynamic spectrum access/cognitive radio wireless networks: a survey," Computer Networks, vol. 50, no. 13, pp. 21272159, 2006.

[12] R. Umar and A. U. H. Sheikh, "A comparative study of spectrum awareness techniques for cognitive radio oriented wireless networks," Physical Communication, vol. 9, pp. 148-170, 2013.

[13] T. Dhope and D. Simunic, "Spectrum sensing algorithm for cognitive radio networks for dynamic spectrum access for IEEE 802.1laf standard," International Journal of Research and Reviews in Wireless Sensor Networks, vol. 2, no. 1, pp. 77-84, 2012.

[14] D. Cabric, S. M. Mishra, and R. W. Brodersen, "Implementation issues in spectrum sensing for cognitive radios," in Proceedings of the Conference Record of the 38th Asilomar Conference on Signals, Systems and Computers, pp. 772-776, Pacific Grove, Calif, USA, November 2004.

[15] A. Ghasemi and E. S. Sousa, "Optimization of spectrum sensing for opportunistic spectrum access in cognitive radio networks," in Proceedings of the 4th Annual IEEE Consumer Communications and Networking Conference, pp. 1022-1026, January 2007.

[16] D. G. Altman and J. M. Bland, "Parametric $v$ non-parametric methods for data analysis," BMJ, vol. 338, Article ID a3167, 2009.

[17] S. Kay, "Detection of a Sinuisoid in White Noise by Autoregressive Spectrum Analysis," Proceeding of the IEEE International Conference on Acoustics, Speech and Signal Processing (ICASSP '80), pp. 658-661, 1980.

[18] P. Stoica and L. M. Randolph, Spectral Analysis of Signals, Pearson/Prentice Hall, Upper Saddle River, NJ, USA, 2005.

[19] A. Gorcin, H. Celebi, K. A. Qaraqe, and H. Arslan, "An autoregressive approach for spectrum occupancy modeling and prediction based on synchronous measurements," in Proceedings of the IEEE 22nd International Symposium on Personal, Indoor and Mobile Radio Communications (PIMRC '11), pp. 705-709, Toronto, Canada, September 2011. 
[20] Z. Wen, T. Luo, W. Xiang, S. Majhi, and Y. Ma, "Autoregressive spectrum hole prediction model for cognitive radio systems," in Proceedings of the IEEE International Conference on Communications Workshops (ICC '08), pp. 154-157, May 2008.

[21] C. Dong, Y. Dong, and L. Wang, "Autoregressive channel prediction model for cognitive radio," in Proceedings of the 5th International Conference on Wireless Communications, Networking and Mobile Computing (WiCOM '09), pp. 1-4, Beijing, China, September 2009.

[22] C. Dong, Y. Dong, L. Wang, Z. Yang, and H. Zhang, "Particle filtering based autoregressive channel prediction model," Journal of Electronics, vol. 27, no. 3, pp. 316-320, 2010.

[23] A. M. Aibinu, M. J. E. Salami, and A. A. Shafie, "Artificial neural network based autoregressive modeling technique with application in voice activity detection," Engineering Applications of Artificial Intelligence, vol. 25, no. 6, pp. 1265-1276, 2012.

[24] P. Welch, "The use of fast fourier transform for the estimation of power spectra: a method based on time averaging over short, modified periodograms," IEEE Transactions on Audio and Electroacoustics, vol. 15, no. 2, pp. 70-73, 1967.

[25] M. Matinmikko, H. Sarvanko, M. Mustonen, and A. Mämmelä, "Performance of spectrum sensing using welch's periodogram in rayleigh fading channel," in Proceedings of the 4th International Conference on Cognitive Radio Oriented Wireless Networks and Communications, 5, p. 1, Hannover, Germany, June 2009.

[26] I. Harjula, A. Hekkala, M. Matinmikko, and M. Mustonen, "Performance evaluation of spectrum sensing using welch periodogram for OFDM signals," in Proceedings of the IEEE 73rd Vehicular Technology Conference (VTC '11), pp. 1-5, May 2011.

[27] N. Wang and G. Yue, "Optimal threshold of Welch's periodogram for sensing OFDM signals at low SNR levels," in Proceedings of the 19th European Wireless Conference (EW), pp. 1-5, Guildford, UK, April 2013.

[28] S. Haykin, "The multitaper method for accurate spectrum sensing in cognitive radio environments," in Proceeding of the 41st Asilomar Conference on Signals, Systems and Computers of the IEEE Conference Record (ACSSC '07), pp. 436-439, Pacific Grove, Calif, USA, November 2007.

[29] J. Wang and Q. T. Zhang, "A multitaper spectrum based detector for cognitive radio," in Proceedings of the IEEE Wireless Communications and Networking Conference (WCNC '09), pp. 1-5, April 2009.

[30] H. Gao, M. Wu, C. Xu, and Q. Wu, "An improved multitaper method for spectrum sensing in cognitive radio networks," in Proceedings of the 3rd IEEE International Conference on Computer Science and Information Technology (ICCSIT '10), pp. 393-396, Chengdu, China, July 2010.

[31] H. Urkowitz, "Energy detection of unknown deterministic signals," Proceedings of the IEEE, vol. 55, no. 4, pp. 523-531, 1967. 

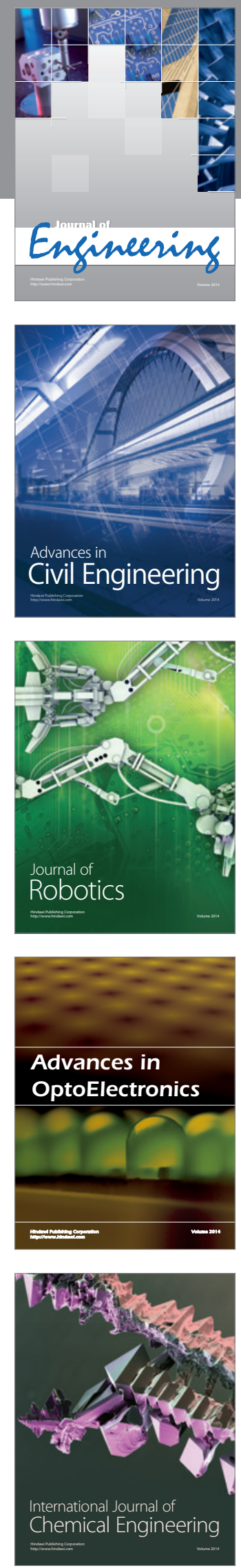

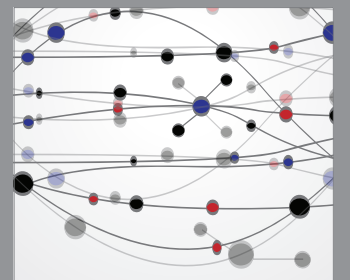

The Scientific World Journal
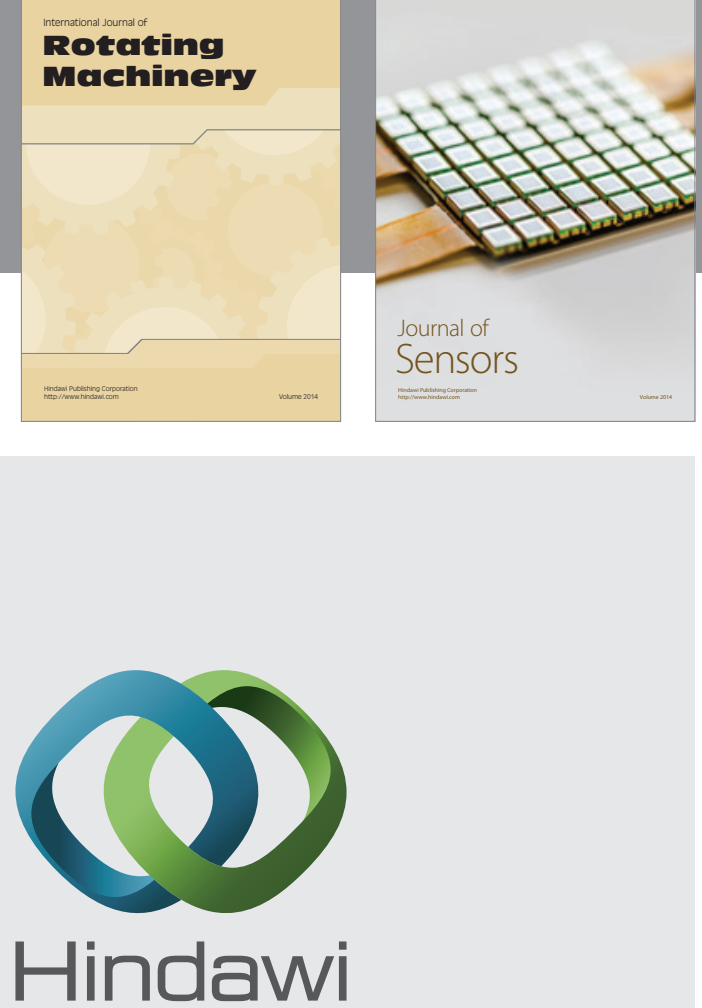

Submit your manuscripts at http://www.hindawi.com
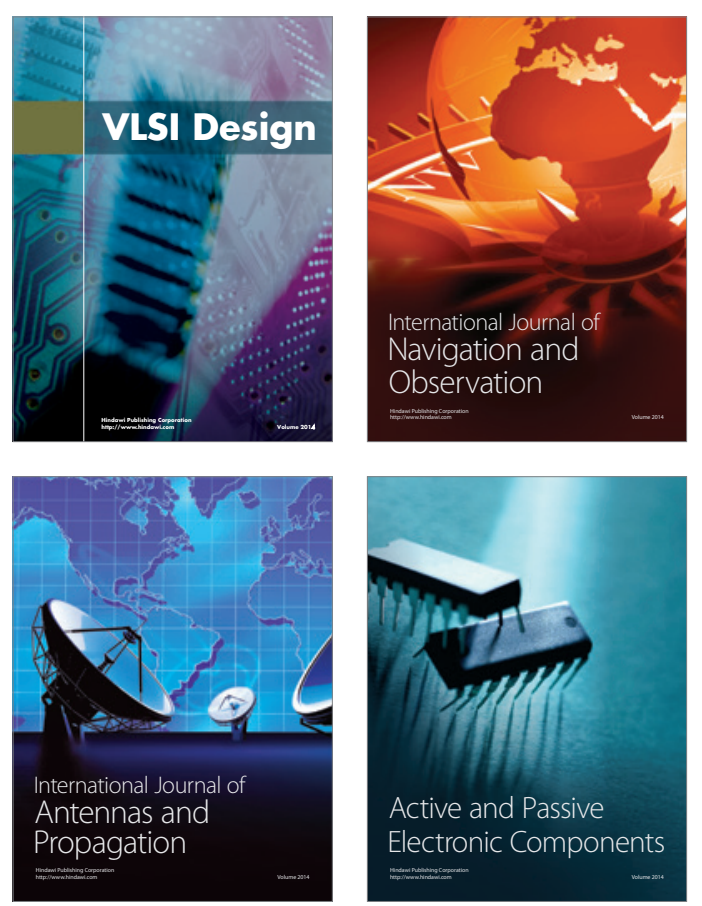
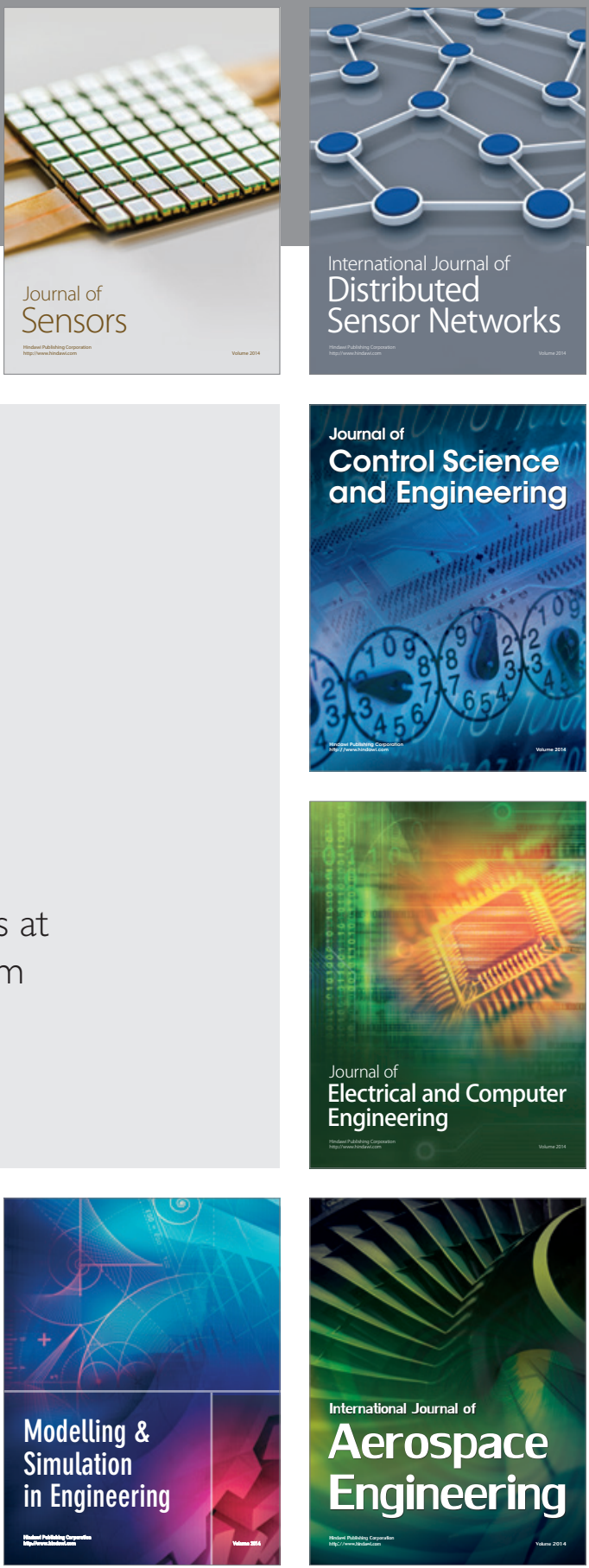

Journal of

Control Science

and Engineering
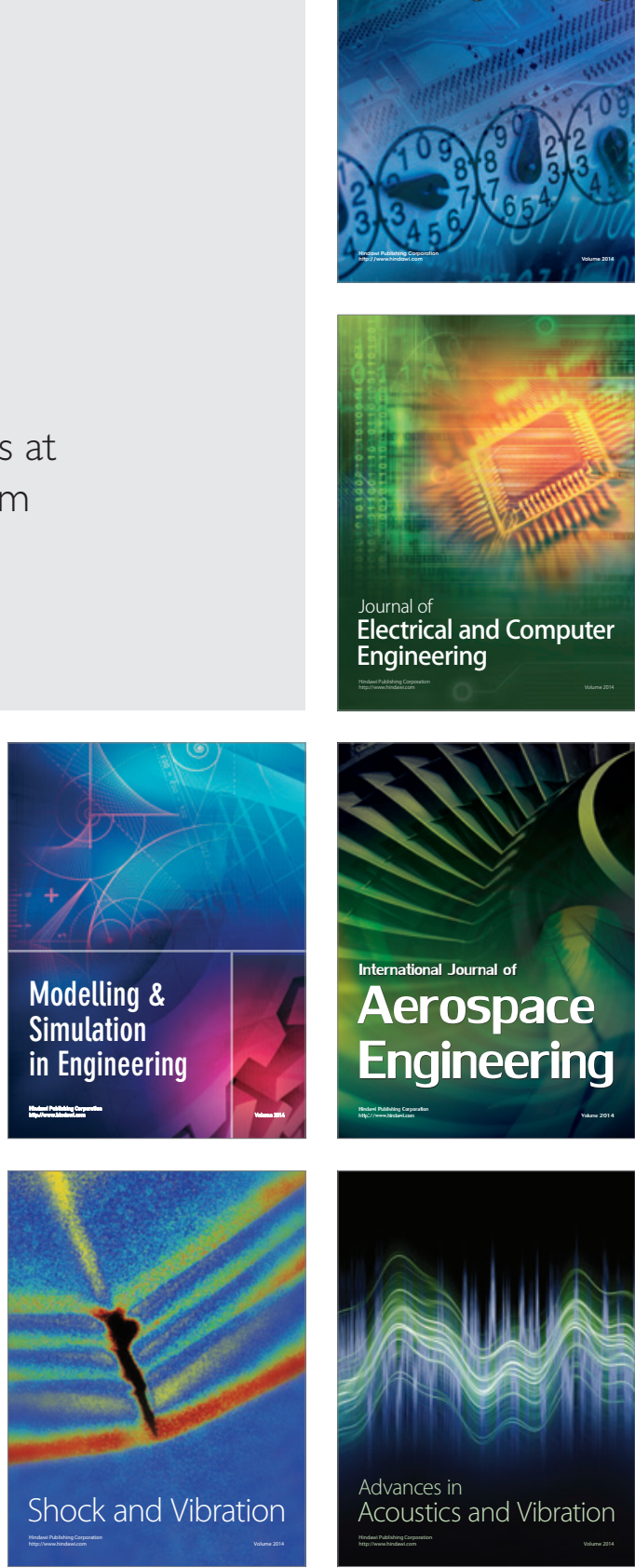